\title{
Competition between [2 + 1]- and [4+1]-cycloaddition mechanisms in reactions of conjugated nitroalkenes with dichlorocarbene in the light of a DFT computational study
}

\author{
Radwan A. Alnajjar ${ }^{1,2} \cdot$ Radomir Jasiński $^{3}$ (B) \\ Received: 9 January 2019 / Accepted: 21 March 2019 / Published online: 11 May 2019 \\ (C) The Author(s) 2019
}

\begin{abstract}
The competition between $[2+1]$ and $[4+1]$ channels regarding reactions of conjugated nitroalkenes with dichlorocarbene was explored based on B3LYP/6-31G(d) calculations. It was found that, in the case of cycloadditions involving parent nitroethene and its 1-substituted analogs, the [2+1] scheme should be treated as possible only from the kinetic process point of view. On the other hand, in similar reactions involving 2 -substituted nitroethenes, both channels considered may compete. Additionally, mechanistic aspects of all cycloadditions were analyzed. It was found that the considered [2+1]-cycloadditions proceed via a non-polar mechanism with a biradicaloidal transition state (TS), whereas $[4+1]$-cycloadditions proceed via a polar mechanism with a zwitterionic TS.
\end{abstract}

Keywords Nitroalkenes $\cdot$ Cycloaddition $\cdot$ Molecular mechanism $\cdot$ DFT study $\cdot$ Molecular electron density theory

\section{Introduction}

Nitro-functionalized carbo- and heterocycles play an important role in modern organic chemistry. This is a consequence of a wide range of further functionalization that may be realized via transformation of nitrocompounds into nitrile $\mathrm{N}$-oxides $[1,2]$, oximes $[3,4]$, hydroxylamines $[5$, 6], nitronates [2, 4], carbonyl compounds [4, 7], aminoalcohols $[8,9]$ and others $[4,10]$; this makes the effective synthesis of many valuable compounds possible.

The most universal methodology for preparing nitrofunctionalized cyclic compounds is cycloaddition reactions involving respective conjugated nitroalkenes (CNA) [4, $11]$. In this way, several four- $[12,13]$, five -[14-17], and

Radomir Jasiński

radomir@chemia.pk.edu.pl

1 Department of Chemistry, Faculty of Science, University of Benghazi, Benghazi, Libya

2 Department of Chemistry, University of Cape Town, Rondebosch 7701, South Africa

3 Department of Organic Chemistry, Cracov University of Technology, Kraków, Poland six-membered [18-21] nitro-cycles have been prepared. This is possible via $[2+2],[3+2]$, and $[4+2]$ cycloadditions, respectively. All these processes have been realized under relatively mild conditions and with full atomic economy. A detailed literature survey shows, unfortunately, [11], that any examples of [2+1]-cycloaddition involving conjugated nitroalkenes (which gave the possibility of the synthesis of nitro-functionalized cyclopropanes) are not known. The work presented here initiates a comprehensive study in this area. In particular, we decided to shed light on the molecular mechanism of model transformations involving a representative series of conjugated nitroalkenes (1ae) and dichlorocarbene (2) (Scheme 1). Carbene $\mathbf{2}$ is well known and may be easily generated in the reaction environment [22-25]. On the other hand, five nitroalkenes (1ae) characterized by a different type of substitution were selected. All these nitrocompounds may be prepared with good yields [26], can exist as relatively stable compounds, and have been recently tested as components of other type cycloadditions (nitroethene 1a [14, 16, 27], 2-niroprop-1ene 1b [28], 1-chloro-1-nitroethene 1c [14, 18, 29], E-1nitroprop-1-ene 1d [28, 30, 31], E-2-chloro-1-nitroethene [31]). We hope that this study will help to further understand the nature of the title reactions and will be useful in organic synthesis. 


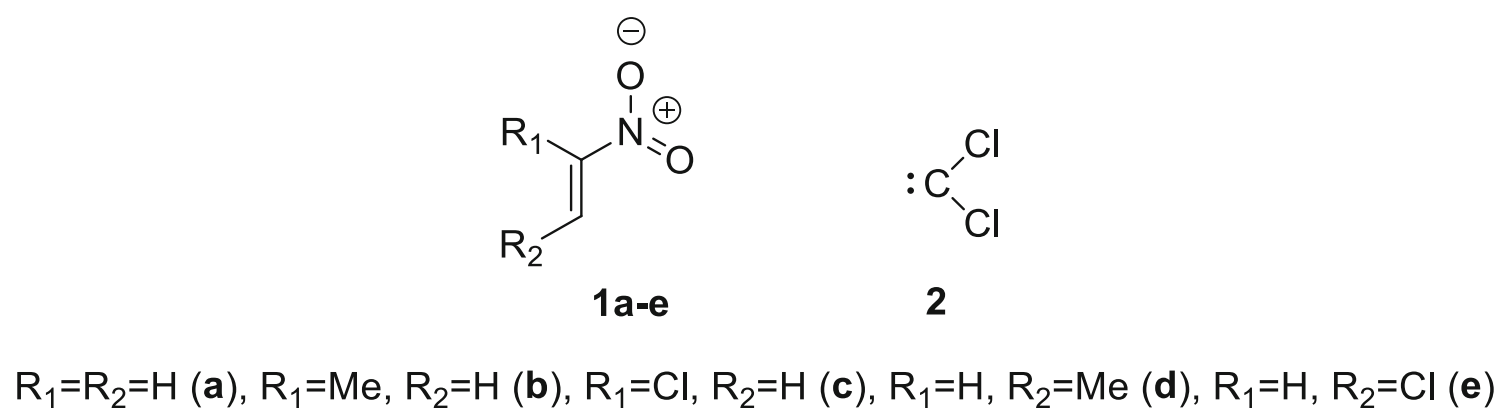

Scheme 1 Structures of selected cycloaddition components

\section{Computational methods}

All quantum-chemical calculations were performed using 'Prometheus' infrastructure shared by the ACK 'Cyfronet' in Kracow, Poland. The B3LYP method is formed through a combination of Becke's three-parameter hybrid functional [32] and the LYP semi-local correlation function [33] with $6-31 \mathrm{G}(\mathrm{d})$ basis set, and was implemented in the Gaussian 09 [34] program. We recently applied an identical level of theory successfully to resolving several different aspects of cycloaddition reactions involving CNAs, such as regioselectivity $[14,17,21,30]$, stereoselectivity $[21,31]$, molecular mechanism [14, 16, 17, 21, 31, 35], solvent effects [21], substituent effects [14, 21, 35], etc. So, it may be assumed that this approach is adequate for analysis of the issues described above. Subsequently, we also examined higher levels of theory for resolving of the title problem. In these calculations, different types of functionals and basis sets were applied. It was found that all these approaches suggest the same kinetic preferences of reaction channels and similar nature of asynchronicity of transition states (TS). This confirms that the B3LYP/6$31 \mathrm{G}(\mathrm{d})$ theory level is adequate for our needs.

All critical structures were optimized using the Berny algorithm [36] and were characterized by frequency calculations. It was found that all addends, molecular complexes (MC), and products (P) had positive Hessian matrices, whereas all TS had one negative eigenvalue in their Hessian matrices. For all TS, intrinsic reaction coordinate (IRC) calculations were performed. The solvent effect was implemented using the polarizable continuum model (PCM) [37]. Global electron density transfer between substructures of the transition state (GEDT) [38] was calculated according to the equation:

$\mathrm{GEDT}=\Sigma \mathrm{q}_{\mathrm{A}}$

where $\mathrm{q}_{\mathrm{A}}$ is the net charge, and the sum is taken over all the atoms of nitroalkene.

Results of quantum chemical calculations are collected in Tables 1, 2, 3, 4, and 5 .

\section{Results and discussion}

The [2+1]-cycloadition (21CA) process involving CNAs 1ae and dichlorocarbene 2 (Scheme 1) formally should be resulted in nitrofunctionalized, cyclopropane derivatives (3a-e respectively). A few, recent theoretical works [39-41] suggest a one-step mechanism for some $[2+1]$-cycloadditions involving dichlorocarbene. On the other hand, earlier reports [42] suggest a stepwise mechanism, with zwitterionic intermediate. It should be additionally underlined that stepwise mechanisms have been recently documented based on comprehensive experimental and quantum-chemical studies regarding a group of different type cycloadditions involving conjugated nitroalkenes [35, 43, 44]. Therefore, neither one-step nor stepwise mechanisms can be excluded a priori for the reactions studied (Scheme 2).

Next, recent reports confirm that, in some cases, CNAs can be accessed in the addition reactions as hetero-analogs of the 1,3-diene under non-catalytic conditions [45-47]. Therefore, the $[4+1]$-cycloaddition (41CA) scheme should also be considered in the analyzed processes (Scheme 3). Finally, we considered six theoretically possible reaction channels (AE) for reactions 1a-e+2 (Schemes 2 and 3).

Firstly, we decided to analyze the nature of intermolecular interactions during title reactions in the framework of molecular electron density theory (MEDT) [48]. For this purpose, global electrophilicity $(\omega)$ for all considered addents were calculated using equations recommended by Parr [49] and Domingo [50, 51]:

Table 1 Key electronic properties of CNAs 1a-e and carbene 2

\begin{tabular}{llll}
\hline Cycloaddition components & $\mu[\mathrm{eV}]$ & $\eta[\mathrm{eV}]$ & $\omega[\mathrm{eV}]$ \\
\hline $\mathbf{1 a}$ & -5.33 & 5.45 & 2.61 \\
$\mathbf{1 b}$ & -5.16 & 5.48 & 2.43 \\
$\mathbf{1 c}$ & -5.47 & 5.11 & 2.93 \\
$\mathbf{1 d}$ & -5.08 & 5.48 & 2.35 \\
$\mathbf{1 e}$ & -5.59 & 5.40 & 2.89 \\
$\mathbf{2}$ & -5.45 & 3.80 & 3.91 \\
\hline
\end{tabular}


Table 2 Kinetic and thermodynamic parameters for 21CA and 41CA of selected conjugated nitroalkenes (1a-e) with dichlorocarbene 2 in different solvents in the light of B3LYP/6-31G(d) calculations ( $\Delta H$ and $\Delta G$ are in kcal mol ${ }^{-1} ; \Delta S$ is in cal mol ${ }^{-1} \mathrm{~K}^{-1}$ )

\begin{tabular}{|c|c|c|c|c|c|c|c|c|}
\hline \multirow{2}{*}{ Nitroalkene } & \multirow{2}{*}{ Solvent } & \multirow{2}{*}{ Transition } & \multicolumn{3}{|c|}{$[2+1]$ cycloaddition (21CA) } & \multicolumn{3}{|c|}{ [4+1] cycloaddition (41CA) } \\
\hline & & & $\Delta \mathrm{H}$ & $\Delta \mathrm{G}$ & $\Delta \mathrm{S}$ & $\Delta \mathrm{H}$ & $\Delta \mathrm{G}$ & $\Delta \mathrm{S}$ \\
\hline \multirow{6}{*}{$1 \mathbf{a}$} & \multirow{3}{*}{ Toluene } & $1 \mathrm{a}+2 \rightarrow \mathrm{MC}$ & & & & -1.11 & 6.55 & -25.71 \\
\hline & & $1 \mathrm{a}+2 \rightarrow \mathrm{TS}$ & 1.49 & 12.77 & -37.84 & 6.46 & 18.32 & -39.75 \\
\hline & & $1 \mathrm{a}+2 \rightarrow \mathrm{P}$ & -56.65 & -43.10 & -45.43 & -68.72 & -54.72 & -46.96 \\
\hline & \multirow{3}{*}{ Nitromethane } & $1 \mathrm{a}+2 \rightarrow \mathrm{MC}$ & & & & -0.28 & 6.98 & -24.37 \\
\hline & & $1 \mathrm{a}+2 \rightarrow \mathrm{TS}$ & 1.75 & 12.93 & -37.51 & 6.33 & 18.40 & -40.48 \\
\hline & & $1 \mathrm{a}+2 \rightarrow \mathrm{P}$ & -56.12 & -42.57 & -45.45 & -68.91 & -55.00 & -46.64 \\
\hline \multirow{6}{*}{$\mathbf{1 b}$} & \multirow{3}{*}{ Toluene } & $1 \mathrm{a}+2 \rightarrow \mathrm{MC}$ & & & & -0.89 & 6.21 & -23.79 \\
\hline & & $1 \mathrm{a}+2 \rightarrow \mathrm{TS}$ & 0.52 & 11.68 & -37.40 & 5.92 & 17.79 & -39.80 \\
\hline & & $1 \mathrm{a}+2 \rightarrow \mathrm{P}$ & -54.28 & -40.62 & -45.80 & -70.47 & -57.06 & -44.99 \\
\hline & \multirow{3}{*}{ Nitromethane } & $1 \mathrm{a}+2 \rightarrow \mathrm{MC}$ & & & & -2.51 & 4.48 & -23.47 \\
\hline & & $1 \mathrm{a}+2 \rightarrow \mathrm{TS}$ & 0.87 & 11.99 & -37.30 & 5.67 & 17.55 & -39.84 \\
\hline & & $1 \mathrm{a}+2 \rightarrow \mathrm{P}$ & -53.72 & -40.06 & -45.81 & -70.93 & -57.57 & -44.83 \\
\hline \multirow{3}{*}{$\mathrm{Cl} \mathrm{NO}_{2}$} & \multirow{3}{*}{ Toluene } & $1 \mathrm{a}+2 \rightarrow \mathrm{MC}$ & & & & -1.09 & 6.22 & -24.54 \\
\hline & & $1 \mathrm{a}+2 \rightarrow \mathrm{TS}$ & 0.29 & 11.47 & -37.50 & 7.40 & 19.25 & -39.74 \\
\hline & & $1 \mathrm{a}+2 \rightarrow \mathrm{P}$ & -52.32 & -38.64 & -45.90 & -68.74 & -54.71 & -47.06 \\
\hline \multirow{3}{*}{ 1c } & \multirow{3}{*}{ Nitromethane } & $1 \mathrm{a}+2 \rightarrow \mathrm{MC}$ & & & & -0.30 & 6.70 & -23.49 \\
\hline & & $1 \mathrm{a}+2 \rightarrow \mathrm{TS}$ & 0.71 & 11.82 & -37.28 & 7.98 & 19.41 & -38.35 \\
\hline & & $1 \mathrm{a}+2 \rightarrow \mathrm{P}$ & -51.70 & -37.97 & -46.04 & -68.59 & -54.68 & -46.66 \\
\hline \multirow{7}{*}{ 1d } & \multirow{3}{*}{ Toluene } & $1 \mathrm{a}+2 \rightarrow \mathrm{MC}$ & -1.22 & 4.53 & -19.31 & -2.50 & 4.39 & -23.11 \\
\hline & & $1 \mathrm{a}+2 \rightarrow \mathrm{TS}$ & 1.58 & 12.53 & -36.74 & 3.41 & 14.86 & -38.39 \\
\hline & & $1 \mathrm{a}+2 \rightarrow \mathrm{P}$ & -54.85 & -41.82 & -43.71 & -64.94 & -51.10 & -46.42 \\
\hline & \multirow{3}{*}{ Nitromethane } & $1 \mathrm{a}+2 \rightarrow \mathrm{MC}$ & -0.72 & 4.91 & -18.87 & -1.62 & 4.93 & -21.95 \\
\hline & & $1 \mathrm{a}+2 \rightarrow \mathrm{TS}$ & 2.03 & 12.89 & -36.45 & 2.92 & 14.32 & -38.23 \\
\hline & & $1 \mathrm{a}+2 \rightarrow \mathrm{P}$ & -54.04 & -41.02 & -43.67 & -64.68 & -50.90 & -46.20 \\
\hline & \multirow{3}{*}{ Toluene } & $1 \mathrm{a}+2 \rightarrow \mathrm{MC}$ & -1.72 & 5.99 & -25.88 & -1.72 & 5.90 & -25.56 \\
\hline \multirow{5}{*}{ 1e } & & $1 \mathrm{a}+2 \rightarrow \mathrm{TS}$ & 4.79 & 16.60 & -39.61 & 5.62 & 17.56 & -40.06 \\
\hline & & $1 \mathrm{a}+2 \rightarrow \mathrm{P}$ & -51.98 & -38.33 & -45.80 & -62.26 & -47.99 & -47.84 \\
\hline & \multirow{3}{*}{ Nitromethane } & $1 \mathrm{a}+2 \rightarrow \mathrm{MC}$ & -0.81 & 6.63 & -24.95 & -0.83 & 6.79 & -25.57 \\
\hline & & $1 \mathrm{a}+2 \rightarrow \mathrm{TS}$ & 5.05 & 16.81 & -39.46 & 5.77 & 17.76 & -40.21 \\
\hline & & $1 \mathrm{a}+2 \rightarrow \mathrm{P}$ & -51.33 & -37.64 & -45.90 & -62.19 & -48.03 & -47.50 \\
\hline
\end{tabular}

$\omega=\mu^{2} / 2 \eta$

$\mu \approx\left(\mathrm{E}_{\text {HOMO }}+\mathrm{E}_{\text {LUMO }}\right) / 2$

$\eta \approx \mathrm{E}_{\text {LUMO }}-\mathrm{E}_{\text {HOMO }}$

As is evident in the light of the data presented in Table 1, in the Domingo scale [50], all considered CNAs should be treated as strongly electrophilic agents. Dichlorocarbene $\mathbf{2}$ should be localized in the same group. However, its global electrophilicity $\omega$ is higher. In particular, the difference between $\omega$ values in the reactions involving nitroethene $\mathbf{1 a}$ and their alkyl-substituted analogs is equally more than $1.3 \mathrm{eV}$. Next, for the similar processes involving chloro-substituted CNAs $(\mathbf{1 c}, \mathbf{e})$, the analogous different is equal about $1 \mathrm{eV}$. So, in the case of all considered reactions, the polar mechanism can be treatment as allowed.

In the next step, we decided to fully explore of reaction channels leading finally to competitive cycloadducts $\mathbf{3}$ and 4. It was found that the energetic profile of model $21 \mathrm{CA}$ $\mathbf{1} \mathbf{a}+\mathbf{2} \rightarrow \mathbf{3}$ a suggests a one-step mechanism of addents transformation in toluene solution (Fig. 1, Table 1). In particular, between valleys of individual reagents and product, only one critical point was detected, which is associated with the existence of TS. Reaching this point in the reaction system required rather a small amount of enthalpy $(\Delta H=$ $1.5 \mathrm{kcal} \mathrm{mol}^{-1}$ ). Subsequently, due to the reduction of the entropy of the reaction system, the Gibbs free energy of the activation is higher than $\Delta H$ and is equal to $12.8 \mathrm{kcal} \mathrm{mol}^{-1}$. Generally, it is a rather low value, which allowed the reaction to proceed at room temperature.

Within the localized TS, two new sigma-bonds are formed: C3-C1 and C2-C1 (Fig. 2, Table 2). The key distances between reaction centers within the TS are, however, distinctly different. In particular, the bond formed with the participation of $\beta$ carbon atom derived from nitrovinyl moiety (2.271 $\AA$ ) is more advanced. The second new bond $(\mathrm{C} 3-\mathrm{C} 1)$ is more than $0.5 \AA$ longer. This suggests that the considered TS may exhibit a polar, zwitterionic nature. However, this hypothesis can be excluded due to the value of GEDT, which is nearly equal to 
Table 3 Key parameters of critical structures for 21CA and 41CA of selected conjugated nitroalkenes (1a-e) with dichlorocarbene 2 in different solvents in the light of B3LYP/6-31G(d) calculations

\begin{tabular}{|c|c|c|c|c|c|c|c|c|}
\hline \multirow{3}{*}{$\begin{array}{l}\text { Nitro- } \\
\text { alkene }\end{array}$} & \multirow{3}{*}{ Solvent } & \multirow{3}{*}{$\begin{array}{l}\text { Struc- } \\
\text { ture }\end{array}$} & \multicolumn{3}{|c|}{$[2+1]$ cycloaddition $(21 \mathrm{CA})$} & \multicolumn{3}{|c|}{ [4+1] cycloaddition (41CA) } \\
\hline & & & \multicolumn{3}{|c|}{$3{ }_{2}^{\mathrm{NO}_{2}},{ }_{1}$} & \multicolumn{3}{|c|}{ 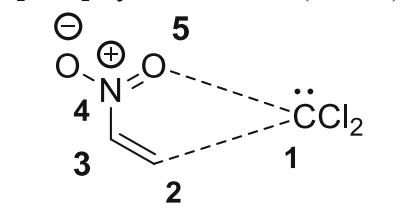 } \\
\hline & & & $\begin{array}{c}\mathrm{r}_{\mathrm{C} 3-\mathrm{C} 1} \\
{[\AA]}\end{array}$ & $\begin{array}{c}\mathrm{r}_{\mathrm{C} 2-\mathrm{C} 1} \\
[\AA]]\end{array}$ & $\begin{array}{c}\text { GEDT } \\
{[\mathrm{e}]}\end{array}$ & $\begin{array}{c}\mathrm{r}_{\mathrm{O} 5-\mathrm{C} 1} \\
{[\AA]}\end{array}$ & $\begin{array}{c}\mathrm{r}_{\mathrm{C} 2-\mathrm{C} 1} \\
{[\AA]}\end{array}$ & $\begin{array}{c}\text { GEDT } \\
{[\mathrm{e}]}\end{array}$ \\
\hline & & $\mathrm{MC}$ & & & & 2.931 & 3.537 & 0.00 \\
\hline & Toluene & TS & 2.861 & 2.271 & -0.01 & 1.790 & 2.641 & 0.15 \\
\hline & & $\mathrm{P}$ & 1.520 & 1.491 & & 1.367 & 1.546 & \\
\hline & & $\mathrm{MC}$ & & & & 2.976 & 3.580 & 0.00 \\
\hline $1 \mathbf{a}$ & Nitromethane & TS & 2.879 & 2.292 & -0.02 & 1.691 & 2.765 & 0.25 \\
\hline & & $\mathrm{P}$ & 1.521 & 1.490 & & 1.363 & 1.545 & \\
\hline & & $\mathrm{MC}$ & & & & 2.934 & 3.583 & 0.00 \\
\hline $\mathrm{OO}_{2}$ & Toluene & TS & 2.901 & 2.331 & 0.04 & 1.769 & 2.670 & 0.16 \\
\hline & & $\mathrm{P}$ & 1.530 & 1.494 & & 1.364 & 1.542 & \\
\hline & & $\mathrm{MC}$ & & & & 2.984 & 3.651 & 0.00 \\
\hline 1b & Nitromethane & TS & 2.911 & 2.350 & 0.04 & 1.700 & 2.763 & 0.24 \\
\hline & & $\mathrm{P}$ & 1.532 & 1.493 & & 1.360 & 1.540 & \\
\hline & & $\mathrm{MC}$ & & & & 2.981 & 3.523 & 0.00 \\
\hline $\mathrm{NO}_{2}$ & Toluene & TS & 2.969 & 2.377 & 0.01 & 1.999 & 2.479 & 0.03 \\
\hline & & $\mathrm{P}$ & 1.504 & 1.501 & & 1.379 & 1.542 & \\
\hline & & $\mathrm{MC}$ & & & & 3.029 & 3.544 & 0.00 \\
\hline 1c & Nitromethane & TS & 2.980 & 2.390 & 0.00 & 2.001 & 2.489 & 0.03 \\
\hline & & $\mathrm{P}$ & 1.505 & 1.498 & & 1.380 & 1.542 & \\
\hline & & $\mathrm{MC}$ & 3.764 & 3.287 & 0.00 & 2.904 & 3.552 & 0.00 \\
\hline $\mathrm{NO}_{2}$ & Toluene & TS & 2.743 & 2.239 & 0.01 & 1.732 & 2.715 & 0.21 \\
\hline & & $\mathrm{P}$ & 1.520 & 1.499 & & 1.369 & 1.555 & \\
\hline & & $\mathrm{MC}$ & 3.778 & 3.795 & 0.00 & 2.954 & 3.603 & 0.00 \\
\hline 1d & Nitromethane & TS & 2.752 & 2.254 & 0.01 & 1.697 & 2.834 & 0.29 \\
\hline & & $\mathrm{P}$ & 1.521 & 1.498 & & 1.365 & 1.554 & \\
\hline $\mathrm{NO}_{2}$ & & $\mathrm{MC}$ & 4.139 & 3.408 & 0.00 & 2.938 & 3.408 & 0.00 \\
\hline & Toluene & TS & 2.773 & 2.199 & -0.05 & 1.827 & 2.626 & 0.12 \\
\hline & & $\mathrm{P}$ & 1.526 & 1.503 & & 1.377 & 1.566 & \\
\hline $\mathrm{Cl}$ & & $\mathrm{MC}$ & 4.170 & 3.427 & 0.00 & 2.986 & 3.430 & 0.00 \\
\hline & Nitromethane & TS & 2.789 & 2.211 & -0.07 & 1.743 & 2.742 & 0.22 \\
\hline $1 \mathrm{e}$ & & $\mathrm{P}$ & 1.527 & 1.502 & & 1.372 & 1.566 & \\
\hline
\end{tabular}

zero. Therefore, the analyzed TS probably has a biradicaloidal character. The IRC calculations connect the energetic maximum of the transition state, directly with the valleys of individual addents and expected product 3a. Due to Jaque and Murray observation $[52,53]$, the shape of IRC profiles can be connected with the degree of TS asynchronicity. The formation of 3a is full exothermic, which excludes the reversible nature of considered transformation. It should be mentioned that all attempts to optimize the critical structures that can be associated with hypothetical mechanisms $\mathbf{B}$ and $\mathbf{C}$ were unsuccessful. Thus, transformation $\mathbf{1 a}+\mathbf{2} \rightarrow \mathbf{3} \mathbf{a}$ is realized via a non-polar one-step mechanism with a highly asynchronous, biradicaloidal TS.
Subsequently, in the similar way, we analyzed the competitive reaction channel of the transformation $\mathbf{1 a}+\mathbf{2} \rightarrow \mathbf{4 a}$. It was found that four critical points exist on the energetic profile of model $41 \mathrm{CA}(\mathbf{1 a}+\mathbf{2} \rightarrow \mathbf{4 a})$. These points can be associated with: individual reagents, pre-reaction MC, TS and reaction product $\mathbf{4 a}$ (Fig. 1, Table 2). The interactions between starting molecules lead, in the first step, to the formation of the pre-reaction complex MC. This does not require an activation barrier, and is associated with a reduction in enthalpy of about $1 \mathrm{kcal} \mathrm{mol}^{-1}$. It should be underlined that, due to the entropy factor, the $\Delta G$ value of MC formation is greater than zero. Therefore, $\mathrm{MC}$ cannot exist at room temperature as a stable intermediate. Within the $\mathrm{MC}$, the distances between reactions centers are evidently longer than the 
Table 4 Selected parameters of transition states for 21CA and 41CA nitroethene 1a with dichlorocarbene 2 in toluene in the light of DFT calculations at different theory levels

\begin{tabular}{|c|c|c|c|c|}
\hline \multirow[t]{2}{*}{ Level of theory } & \multicolumn{2}{|c|}{$[2+1]$ cycloaddition $(21 \mathrm{CA})$} & \multicolumn{2}{|c|}{ A+1] cyc5oaddition (41CA) } \\
\hline & $\mathbf{r}_{\mathrm{C} 3-\mathrm{C} 1}[\AA]$ & $\mathrm{r}_{\mathrm{C} 2-\mathrm{C} 1}[\AA]$ & $\mathrm{r}_{\mathrm{OS}-\mathrm{C} 1}\left[\mathrm{~A}_{\mathbf{2}}-\overline{-}\right.$ & $\mathrm{r}_{\mathrm{C} 2-\mathrm{C} 1}[\AA]$ \\
\hline B3LYP/6-31G(d) & 2.861 & 2.271 & 1.790 & 2.641 \\
\hline B3LYP/6-31G(d,p) & 2.856 & 2.264 & 1.790 & 2.640 \\
\hline B3LYP/6-31 + G(d) & 2.855 & 2.253 & 1.980 & 2.484 \\
\hline B3LYP/6-311G(d) & 2.816 & 2.230 & 1.837 & 2.556 \\
\hline B3LYP/6-311++G(3d2f,3p2d) & 2.827 & 2.247 & 1.777 & 2.679 \\
\hline B97XD-6-311++G(3d2f,3p2d) & 2.690 & 2.170 & 1.729 & 2.615 \\
\hline $\mathrm{M} 062 \mathrm{x} / 6-31+\mathrm{G}(\mathrm{d})$ & 2.750 & 2.223 & 1.755 & 2.596 \\
\hline
\end{tabular}

typical C-C bonds formed in the TS (Fig. 3). Additionally, MC does not exhibit a CT-complex nature. This was confirmed by the GEDT value (0.00e).

Further transformation of the reaction system along the reaction coordinate lead to an energetic maximum. This maximum is associated with the existence of the TS. The transition into TS required an increase in enthalpy of about $6.5 \mathrm{kcal} \mathrm{mol}^{-1}$. In the parallel, due to the reduction of the entropy of the reaction system, the Gibbs free energy of the activation is higher than $\Delta \mathrm{H}$, and is equal to $>18 \mathrm{kcal} \mathrm{mol}^{-1}$. Within localized TS, two new sigma-bonds are formed: O5C1 and C2-C1 (Fig. 3, Table 2).

Interestingly, the asynchronicity of this TS is clearly higher than in the case of the TS of transformation $\mathbf{1 a}+\mathbf{2} \rightarrow \mathbf{3 a}$. In particular, the interatomic distance O5-C1 is $1.8 \AA$, whereas the $\mathrm{C} 2-\mathrm{C} 1$ distance is $0.85 \AA$ longer. The high asynchronicity is accompanied by transfer between TS substructures. This effect is illustrated by GEDT value, which is $0.15 \mathrm{e}$. So, in contrast to the TS of transformation $\mathbf{1 a}+\mathbf{2} \rightarrow \mathbf{3 a}$, the considered TS should be treated as a polar, zwitterionic structure. IRC calculations connect the energetic maximum of the TS directly with the valleys of individual addents and expected product 4a. All attempts to optimize critical structures associated with the hypothetical mechanisms $\mathbf{E}$ and $\mathbf{F}$ were unsuccessful. Thus, the transformation $\mathbf{1 a}+\mathbf{2} \rightarrow \mathbf{4 a}$ was realized via a polar one-step mechanism with a highly asynchronous, zwitterionic TS. Finally, it can be noted that, in the conditions of competition between 21CA and 41CA schemes, the second direct addent transformation should be treated as forbidden from a kinetic point of view $\left(\Delta \Delta \mathrm{G}>5 \mathrm{kcal} \mathrm{mol}^{-1}\right)$.

The introduction of a more polar solvent (nitromethane) to the reaction environment does not change the nature of energetic profiles of competitive reaction paths. The quantitative description of critical points is, however, quite different. In particular, the activation barriers are slightly higher; this, however, did not change the kinetic preferences of possible channels of addents transformation. Within the TS of $\mathbf{1 a}+\mathbf{2} \rightarrow \mathbf{3 a}$ transformation, the advancement of both new sigma bonds

Table 5 Selected parameters of transition states for 21CA and 41CA of selected conjugated nitroalkenes (1f-m) with dichlorocarbene $\mathbf{2}$ in toluene in the light of B3LYP/6-31G(d) calculations

\begin{tabular}{|c|c|c|c|c|c|c|}
\hline \multicolumn{3}{|c|}{$\begin{array}{l}{ }_{\text {Nitroalkege }}^{\ominus} \\
\mathrm{R}_{1} \mathrm{~N}^{+} \mathrm{O}\end{array}$} & \multicolumn{2}{|c|}{ [2+1] cycloaddition (21CA) } & \multicolumn{2}{|c|}{$\left[\begin{array}{l}{[+1]} \\
(1)\end{array}\right.$} \\
\hline $\mathrm{nr}_{2}$ & $\mathrm{R}_{1}$ & $\mathrm{R}_{2}$ & $\overline{\mathbf{2}} \mathrm{r}_{\mathrm{C} 3-\mathrm{C} 1}(\AA)$ & $\mathrm{r}_{\mathrm{C} 2-\mathrm{C} 1}(\AA)$ & $\mathrm{r}_{\mathrm{O}-\mathrm{C} 1}^{\mathbf{3}}\left(\AA_{\mathbf{2}}^{--}\right.$ & $r_{\mathrm{C} 2-\mathrm{C} 1}(\AA)$ \\
\hline 1f & $\mathrm{Ph}$ & $\mathrm{H}$ & 2.516 & 2.046 & 1.876 & 2.591 \\
\hline $1 \mathrm{~g}$ & $\mathrm{~F}$ & $\mathrm{H}$ & 2.876 & 2.324 & 1.755 & 2.642 \\
\hline $1 \mathrm{~h}$ & $\mathrm{Br}$ & $\mathrm{H}$ & 2.983 & 2.425 & 1.916 & 2.538 \\
\hline $1 \mathrm{i}$ & COOMe & $\mathrm{H}$ & 2.951 & 2.331 & 2.187 & 2.527 \\
\hline $1 \mathrm{j}$ & $\mathrm{H}$ & $\mathrm{Ph}$ & 2.639 & 2.158 & 1.737 & 2.832 \\
\hline $1 \mathrm{k}$ & $\mathrm{H}$ & $\mathrm{F}$ & 2.776 & 2.219 & 1.875 & 2.597 \\
\hline 11 & $\mathrm{H}$ & $\mathrm{Br}$ & 2.778 & 2.204 & 1.997 & 2.484 \\
\hline $1 \mathrm{~m}$ & $\mathrm{H}$ & COOMe & 2.783 & 2.168 & 1.840 & 2.613 \\
\hline
\end{tabular}


Scheme 2 Possible mechanism of $[2+1]$ cycloadditions involving $\mathbf{1}$ and $\mathbf{2 a}-\mathbf{e}$

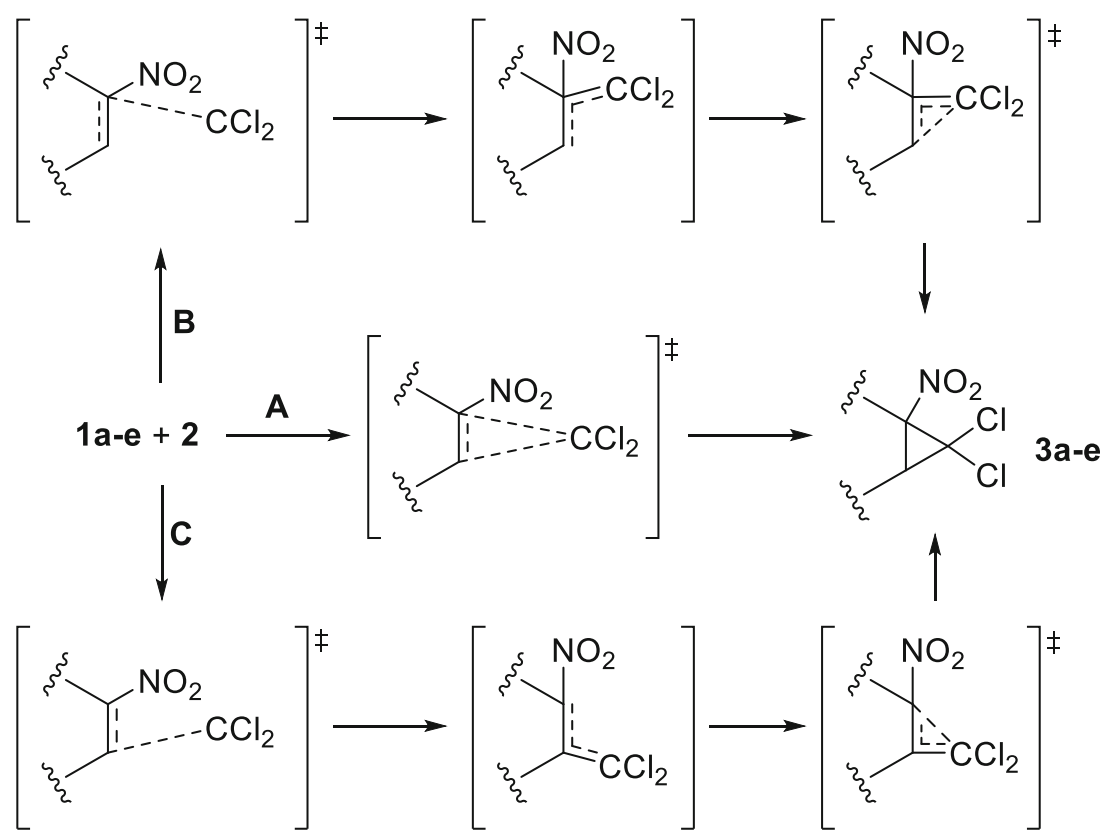

increased similarly. In consequence, the asynchronicity of TS is rather similar as in the case of similar reaction in toluene environment; this is understandable for non-polar reactions and confirms the nature of $\mathbf{1 a}+\mathbf{2} \rightarrow \mathbf{3 a}$ transformation proposed above.

On the other hand, within the TS of $\mathbf{1 a}+\mathbf{2} \rightarrow \mathbf{4 a}$ transformation, the sigma bond $\mathrm{O} 5-\mathrm{C} 1$ is formed faster than in the case of reaction in toluene, whereas the sigma bond $\mathrm{C} 2-\mathrm{C} 1$ is formed more slowly than in the case of the reaction in toluene. As a consequence, the asynchronicity of TS is higher than in the case of the analogous reaction in a toluene environment, but not enough to enforce a stepwise mechanism. The observed influence of solvent polarity on the synchronicity of the TS structure, however, confirms the polar nature of the $\mathbf{1 a}+\mathbf{2} \rightarrow \mathbf{4 a}$ transformation.

Analogously, the competitive 21CA and 41CA processes involving substituted nitroethenes $\mathbf{1 b}$-e were also explored. It was found that, in all considered cases, the molecular mechanism of addent transformation was very similar to the case of the $\mathbf{1 a}+\mathbf{2}$ reaction. Interestingly, the kinetic preference of possible cycloaddition channels is notably changed to some degree. In particular, reactions involving 2 -substituted nitroethenes $\mathbf{2 d}, \mathbf{e}$, the $41 \mathrm{CA}$ scheme may really compete with the $21 \mathrm{CA}$ process. As a
Scheme 3 Possible mechanism of $[4+1]$ cycloadditions involving $\mathbf{1}$ and $\mathbf{2 a}-\mathbf{e}$

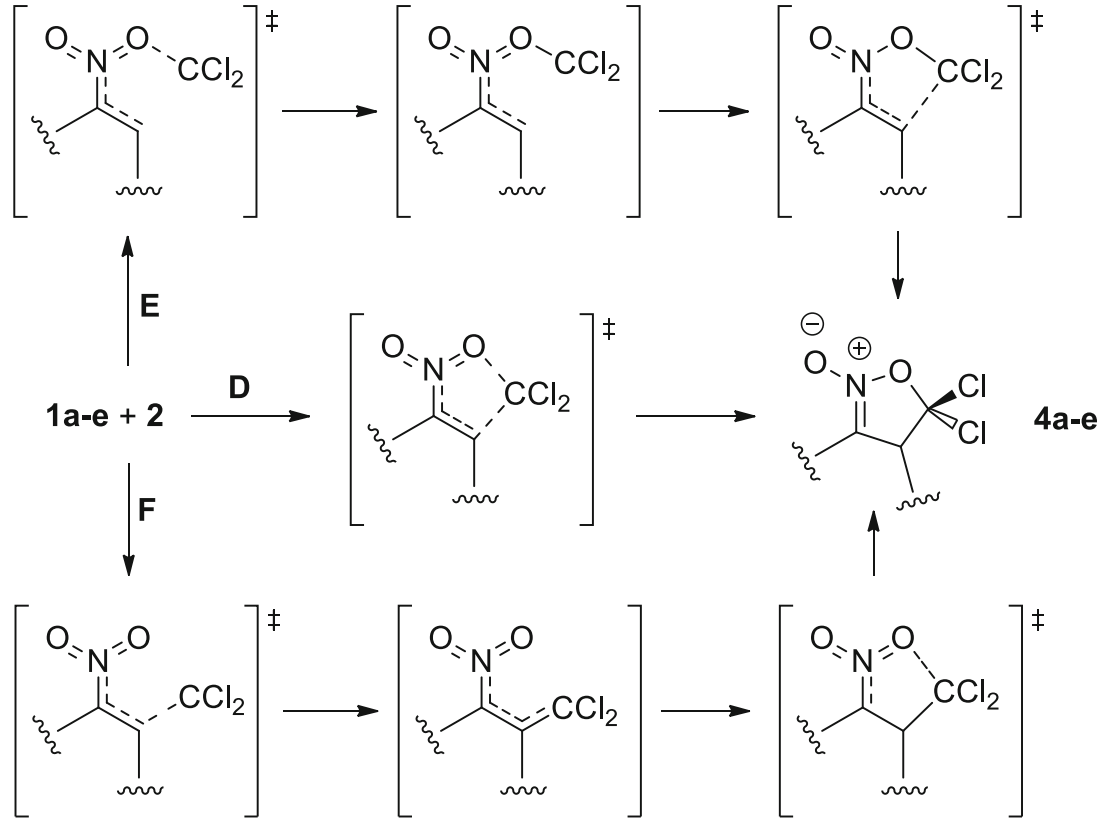


$\Delta \mathrm{H}$

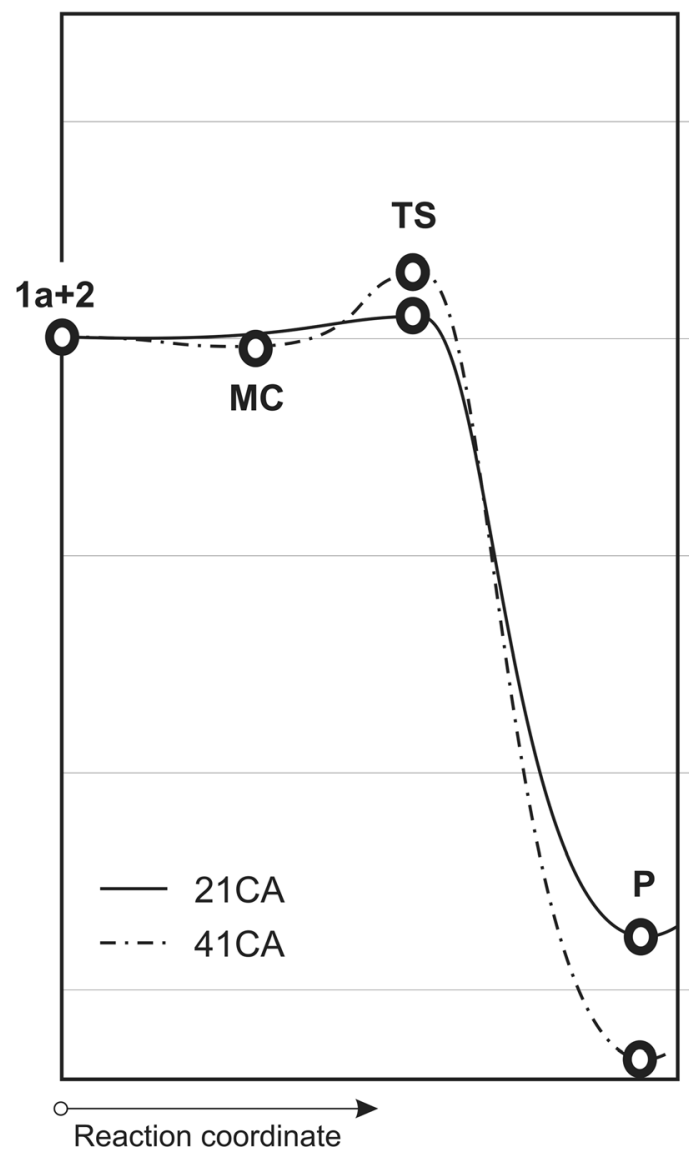

$\Delta \mathrm{G}$

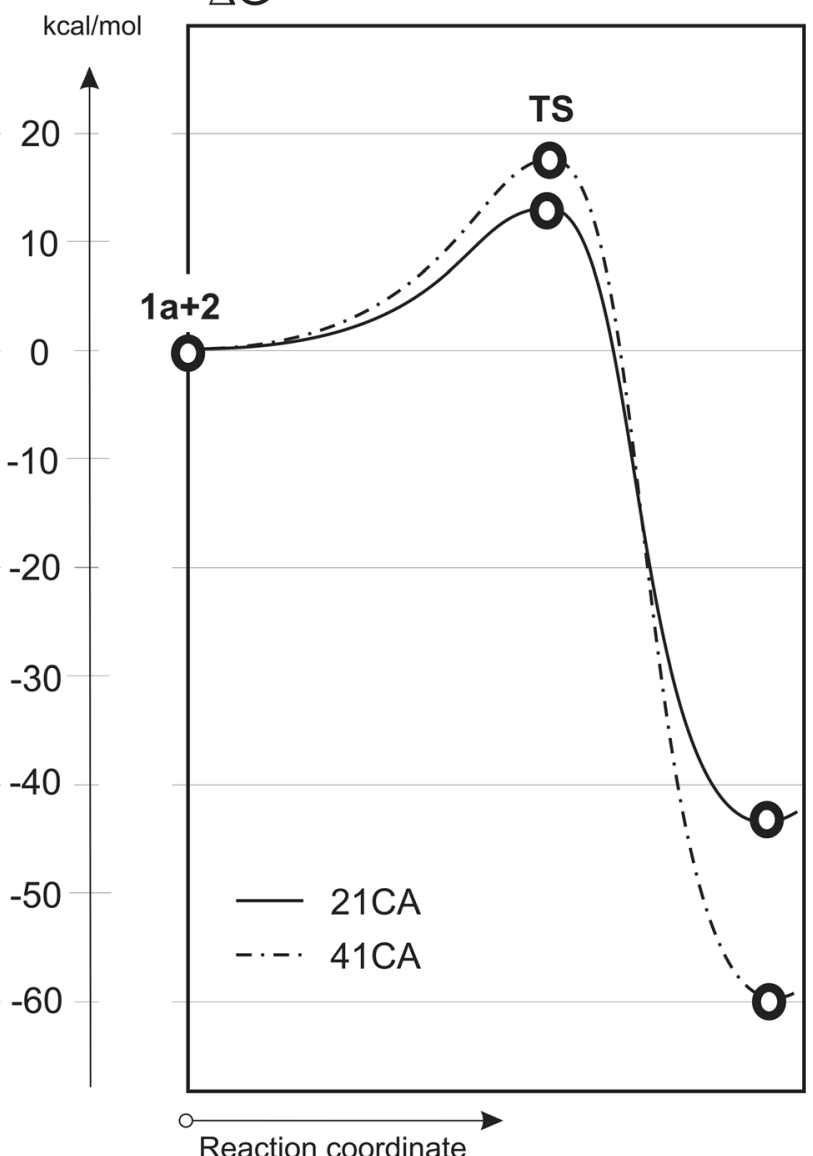

Fig. 1 Reaction profiles for 21CA and 41CA of nitroethene (1a) with dichlorocarbene 2 in toluene solution in the light of B3LYP/6-31G(d) calculations

consequence, within these reactions, two different cycloaddition products (respective $[2+1]$ and $[4+1]$ cycloadducts) may form, with some preference for the $[2+1]$ pathway. So, the process analyzed may be considered as an exciting alternative to known, generally complicated, protocols [54-56] for synthesis of five-membered internal nitronates.

Finally, we also explored in similar way some other reaction systems, including different type substituents at the $\mathrm{C} 1$ or $\mathrm{C} 2$ position of nitroalkene (Table 5). It was found that the molecular mechanism of all of the explored reactions is very similar. So, the proposed reaction scheme can be assumed as the general mechanism for the vast range of cycloaddition reactions between conjugated nitroalkenes and dichlorocarbene.

\section{Conclusions}

DFT investigations at the B3LYP/6-31G(d) level show that molecular mechanisms of competitive [2+1] and [4+1] cycloadditions of conjugated nitroalkenes with dichlorocarbene are substantially different. In particular, all considered [2+1]cycloadditions proceed via a non-polar mechanism with a
Fig. 2 View of key structures for 21CA of nitroethene (1a) with dichlorocarbene $\mathbf{2}$ in toluene solution in the light of B3LYP/6$31 \mathrm{G}(\mathrm{d})$ calculations
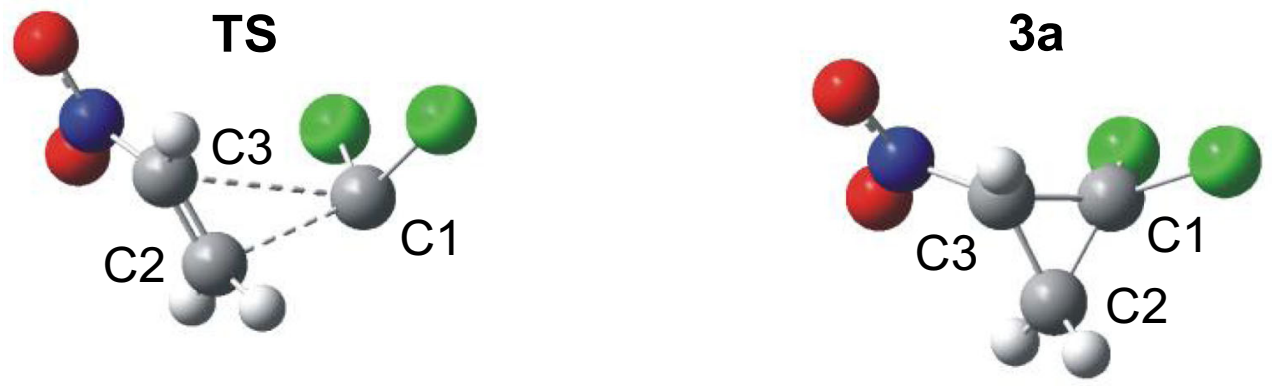

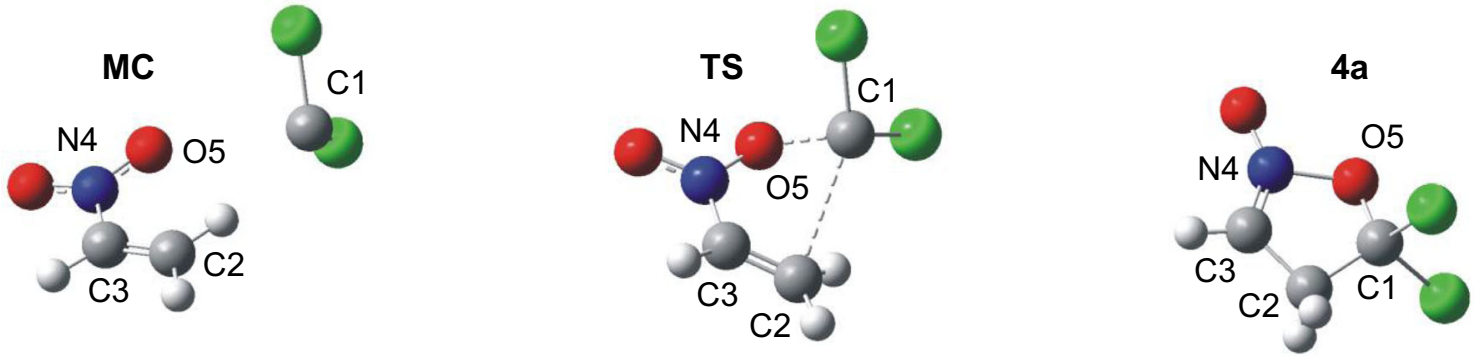

Fig. 3 View of key structures for 41CA of nitroethene (1a) with dichlorocarbene 2 in toluene solution in the light of B3LYP/6-31G(d) calculations

biradicaloidal TS, whereas [4+1]-cycloadditions proceed via a polar mechanism witha zwitterionic TS. Subsequently, we established that the competition between mentioned cycloaddition schemes is possible only in the case of reactions involving 2-substituted nitroethene analogs. In the case of reactions involving a parent nitroethene as well as its 1-substituted analogs, the $[4+1]$ cycloaddition scheme should be treated as forbidden from a kinetic point of view. From a practical point of view, our study suggests that the analyzed processes can be realized under mild conditions, and are a convenient way to prepare nitro-substituted cyclopropane derivatives and (in some cases) five-membered nitronates, which are difficult to realize using other preparation methods.

Acknowledgment The regional computer center "Cyfronet" in Krakow ('Prometheus' cluster, project 'Heterocycles') is thanked for the allocation of computing time.

Open Access This article is distributed under the terms of the Creative Commons Attribution 4.0 International License (http:// creativecommons.org/licenses/by/4.0/), which permits unrestricted use, distribution, and reproduction in any medium, provided you give appropriate credit to the original author(s) and the source, provide a link to the Creative Commons license, and indicate if changes were made.

\section{References}

1. Ajay Kumar K et al (2013) Nitrile oxides: a key intermediate in organic synthesis. IJPCBS 3:91-101

2. Belenkii L (2007) Nitrile oxides. In: Feuer H (ed) Nitrile oxides, nitrone and nitronates in orgnanic synthesis. Wiley, Hoboken, pp 1128

3. Hanson J, Premuzic E (1967) Applications of chromous chlorideII: the reduction of some steroidal nitro-compounds. Tetrahedron 23(10):4105-4110

4. Ono N (2003) The nitro group in organic synthesis, vol 9. Wiley, New York

5. Kende AS, Mendoza JS (1991) Controlled reduction of nitroalkanes to alkyl hydroxylamines or amines by samarium diiodide. Tetrahedron Lett 32(14):1699-1702

6. Rahaim RJ, Maleczka RE (2005) Pd-catalyzed silicon hydride reductions of aromatic and aliphatic nitro groups. Org Lett 7(22): $5087-5090$
7. Selvi T, Srinivasan K (2014) Boron trifluoride mediated ringopening reactions of trans-2-Aryl-3-nitro-cyclopropane-1, 1dicarboxylates. Synthesis of aroylmethylidene malonates as potential building blocks for heterocycles. J Org Chem 79(8):3653-3658

8. Bergmeier SC (2000) The synthesis of vicinal amino alcohols. Tetrahedron 56(17):2561-2576

9. Soengas RG, Silva AM (2012) Indium-catalyzed Henry-type reaction of aldehydes with bromonitroalkanes. Synlett 23(6):873-876

10. Paul B et al (2017) Tandem transformation of nitro compounds into N-methylated amines: greener strategy for the utilization of methanol as a methylating agent. ChemSusChem 10(11):2370-2374

11. Łapczuk-Krygier A, Kącka-Zych A, Kula K (2019) Recent progress in the field of cycloaddition reactions involving conjugated nitroalkenes. Current Chem Lett 8(1):13-38

12. Mohr L-M, Bach T (2017) Intermolecular [2+ 2] photocycloaddition of $\beta$-Nitrostyrenes to olefins upon irradiation with visible light. Synlett 28(20):2946-2950

13. Albrecht $€$ et al (2012) Asymmetric organocatalytic formal [2+2]cycloadditions via bifunctional $\mathrm{H}$-bond directing dienamine catalysis. J Am Chem Soc 134(5):2543-2546

14. Jasiński R, Jasińska E, Dresler E (2017) A DFT computational study of the molecular mechanism of [3+2] cycloaddition reactions between nitroethene and benzonitrile N-oxides. J Mol Model 23(1): 13

15. Mirosław B et al (2018) Regiospecific formation of the nitromethylsubstituted 3-phenyl-4, 5-dihydroisoxazole via [3+2] cycloaddition. Monatsh Chem 149(10):1877-1884

16. Jasiński R (2015) In the searching for zwitterionic intermediates on reaction paths of [3+2] cycloaddition reactions between 2,2,4,4tetramethyl-3-thiocyclobutanone S-methylide and polymerizable olefins. RSC Adv 5(122):101045-101048

17. Jasiński R, Mróz K, Kącka A (2016) Experimental and theoretical DFT study on synthesis of sterically crowded $2,3,3,4) 5$ Tetrasubstituted-4-nitroisoxazolidines via 1, 3-dipolar cycloaddition reactions between Ketonitrones and conjugated Nitroalkenes. J Heterocyclic Chem 53(5):1424-1429

18. Jasiński R (2017) One-step versus two-step mechanism of DielsAlder reaction of 1-chloro-1-nitroethene with cyclopentadiene and furan. J Mol Graph Model 75:55-61

19. Łapczuk-Krygier A, Jasiński R (2014) The crystal structure of (1RS, 4RS, 5RS, 6SR)-5-cyano-5-nitro-6-phenyl-bicyclo [2.2.1] hept-2-ene. Crystallogr Rep 59(7):961-963

20. Jasiński R (2016) First example of stepwise, zwitterionic mechanism for bicyclo [2.2.1] hept-5-ene (norbornene) formation process catalyzed by the 1-butyl-3-methylimidazolium cations. Monatsh Chem 147(7):1207-1213

21. Jasiński R (2018) $\beta$-Trifluoromethylated nitroethenes in DielsAlder reaction with cyclopentadiene: a DFT computational study. J Fluor Chem 206:1-7

22. Khlebnikov A et al (1990) 1, 3-dipolar cycloaddition reactions of ylides formed from pyridines and dichlorocarbene. Chem Heterocycl Compd 26(3):304-311 
23. Rajendran V, Wang M-L (2008) Dichlorocarbene addition to allyl phenyl ether under phase-transfer catalysis conditions - a kinetic study. J Mol Catal A Chem 288(1-2):23-27

24. Egorova AY, Sedavkina VA, Timofeyeva ZY (2000) Interaction of 1, 5-substituted pyrrolin-2-ones with dichlorocarbene under phase transfer catalysis conditions. Molecules 5(10):1082-1084

25. Mohamadi F, Still WC (1986) Dichlorocarbene cyclopropanation of allylic alcohols. Tetrahedron Lett 27(8):893-896

26. Novikov S, et al (1974) Khimiya alifaticheskikh i alitsiklicheskikh nitrosoedinenii [Chemistry of Aliphatic and Alicyclic Nitro Compounds]. Khimiya, Moscow, pp 239

27. Kula K, Łapczuk-Krygier A (2018) A DFT computational study on the $[3+2]$ cycloaddition between parent thionitrone and nitroethene. Current Chem Lett 7(1):27-34

28. Jasiński R (2015) Synthesis and properties of azoles and their derivatives. Part 70. Nitroallylic systems in [2+3] cycloaddition reactions with nitrones: a DFT computational study. J Heterocyclic Chem 52(1):185-192

29. Jasiński R (2015) A stepwise, zwitterionic mechanism for the 1, 3dipolar cycloaddition between (Z)-C-4-methoxyphenyl-Nphenylnitrone and gem-chloronitroethene catalysed by 1-butyl-3methylimidazolium ionic liquid cations. Tetrahedron Lett 56(3): $532-535$

30. Jasiński R et al (2014) Regio-and stereoselectivity of polar [2+ 3] cycloaddition reactions between (Z)-C-(3, 4, 5-trimethoxyphenyl)$\mathrm{N}$-methylnitrone and selected (E)-2-substituted nitroethenes. Open Chem 12(5):586-593

31. Jasiński R (2012) Exploration of regiospecificity phenomenon in $[2+3]$ cycloaddition reactions between arylnitrones and transsubstituted nitroethenes on the basis of the reactivity indices theory. Curr Chem Lett 1(4):157-162

32. Becke AD (1993) Density-functional thermochemistry. III. The role of exact exchange. J Chem Phys 98(7):5648-5652

33. Lee C, Yang W, Parr RG (1988) Development of the Colle-Salvetti correlation-energy formula into a functional of the electron density. Phys Rev B 37(2):785

34. Frisch M et al (2009) Gaussian 09 software. Gaussian Inc, Wallingford

35. Jasiński R et al (2017) A full regioselective and stereoselective synthesis of 4-Nitroisoxazolidines via stepwise [3+2] cycloaddition reactions between (Z)-C-(9-Anthryl)-N-arylnitrones and (E)-3, 3, 3-Trichloro-1-nitroprop-1-ene: comprehensive experimental and theoretical study. J Heterocyclic Chem 54(6):3314-3320

36. Peng $\mathrm{C}$ et al (1996) Using redundant internal coordinates to optimize equilibrium geometries and transition states. J Comput Chem 17(1):49-56

37. Cossi $\mathrm{M}$ et al (2003) Energies, structures, and electronic properties of molecules in solution with the C-PCM solvation model. J Comput Chem 24(6):669-681

38. Domingo LR (2014) A new C-C bond formation model based on the quantum chemical topology of electron density. RSC Adv 4(61):32415-32428

39. Petrushenko IK (2014) [2+1] cycloaddition of dichlorocarbene to finite-size graphene sheets: DFT study. Monatsh Chem 145(6):891896

40. Zeroual A, Benharref A, El Hajbi A (2015) Theoretical study of stereoselectivity of the $[1+2]$ cycloaddition reaction between (1S, 3R, 8S)-2, 2-dichloro-3, 7, 7, 10-tetramethyltricyclo [6, 4, 0 , 0 1.3] dodec-9-ene and dibromocarbene using density functional theory (DFT) B3LYP/6-31G*(d). J Mol Model 21(3):44

41. Lu X, Tian F, Zhang Q (2003) The [2+ 1] cycloadditions of dichlorocarbene, silylene, germylene, and oxycarbonylnitrene onto the sidewall of armchair $(5,5)$ single-wall carbon nanotube. J Phys Chem B 107(33):8388-8391

42. Von E, Doering W, Henderson Jr WA (1958) The electron-seeking demands of dichlorocarbene in its addition to olefins. J Am Chem Soc 80(19):5274-5277

43. Jasiński $R$ et al (2017) Unexpected course of reaction between (E)2-aryl-1-cyano-1-nitroethenes and diazafluorene: why is there no 1 , 3-dipolar cycloaddition? Monatsh Chem 148(5):909-915

44. Jasiński R (2018) Competition between one-step and two-step mechanism in polar [3+ 2] cycloadditions of $(Z)-C-(3,4,5-$ trimethoxyphenyl)-N-methyl-nitrone with (Z)-2-EWG-1-bromo-1nitroethenes. Comput Theor Chem 1125:77-85

45. Jasiński R et al (2014) An experimental and theoretical study of the hetero Diels-Alder reactions between (E)-2-aryl-1-cyano-1nitroethenes and ethyl vinyl ether: one-step or zwitterionic, twostep mechanism? React Kinet Mech Catal 113(2):333-345

46. Tohda $Y$ et al (1988) Synthesis and a novel fragmentation of 6Alkoxy-5, 6-dihydro-4 H-1, 2-oxazine 2-oxide. Bull Chem Soc Jpn 61(2):461-465

47. Jasiński R (2014) Searching for zwitterionic intermediates in hetero diels-alder reactions between methyl $\alpha$, p-dinitrocinnamate and vinyl-alkyl ethers. Comput Theor Chem 1046:93-98

48. Domingo LR, Ríos-Gutiérrez M, Pérez P (2016) Applications of the conceptual density functional theory indices to organic chemistry reactivity. Molecules 21(6):748

49. Parr RG, Szentpaly Lv, Liu S (1999) Electrophilicity index. J Am Chem Soc 121(9):1922-1924

50. Pérez P et al (2003) Quantitative characterization of the global electrophilicity pattern of some reagents involved in 1,3-dipolar cycloaddition reactions. Tetrahedron 59(17):3117-3125

51. Domingo LR, Chamorro E, Pérez P (2008) Understanding the reactivity of captodative ethylenes in polar cycloaddition reactions. A theoretical study. J Org Chem 73(12):4615-4624

52. Yepes D et al (2014) Complementarity of reaction force and electron localization function analyses of asynchronicity in bond formation in Diels-Alder reactions. Phys Chem Chem Phys 16(14): 6726-6734

53. Murray JS et al (2015) Insights into some Diels-Alder cycloadditions via the electrostatic potential and the reaction force constant. Comput Theor Chem 1053:270-280

54. Ioffe S et al (1973) Silylation of nitro-compounds. 3. Dinitrocarbene formation in trimethylsilylation of trinitromethane derivatives. Zh Org Khim 9(5):905-912

55. Kunetsky RA et al (2003) New approach for the synthesis of isoxazoline-N-oxides. Org Lett 5(25):4907-4909

56. Kunetsky RA et al (2006) General method for the synthesis of isoxazoline $\mathrm{N}$-oxides from aliphatic nitro compounds. Synthesis 2006(13):2265-2270

Publisher's note Springer Nature remains neutral with regard to jurisdictional claims in published maps and institutional affiliations. 